\title{
On Singularities of Certain Non-linear Second-Order Ordinary Differential Equations
}

\author{
Galina Filipuk@ and Thomas Kecker
}

\begin{abstract}
The method of blowing up points of indeterminacy of certain systems of two ordinary differential equations is applied to obtain information about the singularity structure of the solutions of the corresponding non-linear differential equations. We first deal with the so-called Painlevé example, which passes the Painlevé test, but the solutions have more complicated singularities. Resolving base points in the equivalent system of equations we can explain the complicated structure of singularities of the original equation. The Smith example has a solution with non-isolated singularity, which is an accumulation point of algebraic singularities. Smith's equation can be written as a system in two ways. We show that the sequence of blow-ups for both systems can be infinite. Another example that we consider is the Painlevé-Ince equation. When the usual Painlevé analysis is applied, it possesses both positive and negative resonances. We show that for three equivalent systems there is an infinite sequence of blow-ups and another one that terminates, which further gives a Laurent expansion of the solution around a movable pole. Moreover, for one system it is even possible to obtain the general solution after a sequence of blow-ups.
\end{abstract}

Mathematics Subject Classification. Primary 34M35, Secondary 14E05, $14 \mathrm{E} 15$.

\section{Introduction}

Since the work of Sophia Kowalevskaya on the rotation of a rigid body around a fixed point [14] it has been realised that the singularity structure of the solutions of a differential equations in the complex plane is an important tool to 
examine the integrability of an equation. The solutions of a given non-linear second-order ODE in general have infinitely many singularities in the complex plane, the location of which, apart from a finite number of fixed singularities of the equation, depends on the initial data of the equation. These singularities are therefore called movable. The behaviour of the solutions at the fixed singularities of an equation can be completely understood, whereas the nature of the movable singularities is less obvious. For second-order equations, essential singularities, branch points (logarithmic or algebraic) and transcendental singularities can in general occur. For higher-order equations, non-isolated singularities can occur, for example the movable barriers in the third-order Chazy equation. In recent years, certain classes of ordinary differential equations (ODEs) in the complex plane were studied which exhibit movable algebraic singularities, that is, locally the solutions can be expressed in terms of convergent Puiseux series expansions. This is a generalisation of the Painlevé property which demands that all movable singularities of the equation be poles. The Painlevé equations are six non-linear second-order ODEs and were discovered by Painlevé and others at the start of the 20th century in the classification of all second-order rational ODEs with this property. They have been extensively studied since by both applied and pure mathematicians, and their solutions, the Painlevé transcendents, have found their entry in the list of special functions. Okamoto has studied all six Painlevé equations in their Hamiltonian form from a geometric point of view in [15], where he introduced the notion of the space of initial conditions, obtained by blowing up the phase space at a finite sequence of points. A blow-up is a construction originating in algebraic geometry to regularise an algebraic curve. It can be adapted to the setting of differential equations where it serves to regularise a system of equations at points of indeterminacy of the equations. The blow-ups where performed somewhat more explicitly by Duistermaat and Joshi [4] for the first Painlevé equation and by Howes and Joshi [11] for the second Painlevé equation.

In this article, we will apply the method of blowing up the phase space to some non-linear second-order differential equations with a more general singularity structure than imposed by the Painlevé property. Although the geometric picture is not as nice as in the Painlevé case, where the solutions of the equations uniformly foliate the space of initial conditions, a great deal of insight can be gained about the singularity structure of the solutions of these equations. Here it is of general interest what types of singularities a solution of an equation can develop when analytically continued in the complex plane. We will consider several examples. The first example is an equation proposed by Painlevé, for which the general solution has, apart from simple poles, also logarithmic branch points as singularities. This behaviour, however, cannot be picked up by Painlevé analysis which only detects the poles of the solutions (the Painlevé example thus passes the Painlevé test). By the method used below, however, we will be able to detect both types of singularities. We resolve two base points in a finite number of steps in the equivalent system of equations 
and this leads to the explanation of the complicated structure of singularities of the original equation. Another example is an equation proposed by Smith [19]. The equation is interesting since although all movable singularities of any solution that can be reached by analytic continuation along a finite length curve are algebraic branch points, however, Smith has given solutions with a singularity that is an accumulation point of algebraic branch points (nonisolated singularity). Such a singularity itself is not algebraic and can only be obtained by analytic continuation along an infinite length curve. As we will see, such complicated behaviour is most likely reflected in the fact that the sequence of blow-ups for this equation does not terminate. Smith's equation can be written as a system in two ways. We show that the sequence of blow-ups for both systems can be infinite. Although we have no proof that the sequence of blow-ups does not terminate, there is a pattern emerging that leads us to believe that this may in fact be the case. Moreover, we show how the algebraic expansions arise from one of the systems. Another example that we consider is the Painlevé-Ince equation. When the usual Painlevé analysis is applied to it, it is easy to verify that it possesses both positive and negative resonances. We show that for three equivalent systems there is an infinite sequence of blow-ups and another one that terminates, which further gives a Laurent expansion of the solution around a movable pole. Moreover, for one system it is even possible to obtain the general meromorphic solution after a sequence of blow-ups. We conclude the paper with a discussion and some more examples that we plan to discuss in more detail in the future. In addition, we will review formally the general method given in [13] for obtaining the singularities of a secondorder differential equation, or, more generally, of a system of two first-order equations, using the method of blowing up the space of dependent variables. This can be seen as an algorithm to detect all different types of singularities in the complex plane that can arise for a given equation, if the sequence of blowups terminates. In the cases where it does not terminate, this should be seen as an indicator that the equation may possess a very complicated singularity structure as in the example of Smith's equation, and a more detailed analysis is needed. In the discussion section we also mention the Hayman equation, which has entire solutions and where we observe infinite sequences of blow-ups.

\section{Regularising Bi-rational Transformations}

The Painlevé equations were discovered in the search for differential equation where the only movable singularities are poles. Painlevé himself classified all equations in the class of second-order rational differential equations $y^{\prime \prime}=R\left(z, y, y^{\prime}\right)$ with this property. He did so by first exploiting necessary criteria for an equation to have this property to eliminate certain types of equations. One such necessary criterion is to pass what is now known as the 
Painlevé test. Inserting a formal Laurent series into the equation, after determining the leading order of a possible solution, one can recursively compute the coefficients of the series. If there is no obstruction in computing the coefficients and a sufficient number of such formal Laurent series solutions exists, the equation is said to pass the test. The result of this classification is that, apart from equations which can be solved in terms of formerly known functions, there are only 6 different types of equations the solutions of which define new transcendental functions, known as the Painlevé transcendents. To prove that these 6 equations in fact possess the Painlevé property requires somewhat more work. In certain proofs for the Painlevé property of the Painlevé equations (e.g. $[10,16]$ ) a main part is played by a system of equations in certain transformed coordinates where the system becomes regular at points where the original variables tend to infinity. For example, for the second Painlevé equation,

$$
y^{\prime \prime}=2 y^{3}+z y+\alpha, \quad \alpha \in \mathbb{C},
$$

the changes of variables

$$
y=u^{-1}, \quad y^{\prime}=\mp\left(u^{-2}+z / 2+(\alpha \pm 1 / 2) u-u^{2} v\right)
$$

lead to the following regular systems of equations at points where $y \rightarrow \infty$, i.e. $u \rightarrow 0$ :

$$
\begin{aligned}
& u^{\prime}= \pm\left(1+z u^{2} / 2+(\alpha \pm 1 / 2) u^{3}-u^{4} v\right) \\
& v^{\prime}= \pm\left(z / 2+(\alpha \pm 1 / 2) u-u^{2} v\right)(\alpha \pm 1 / 2-2 u v) .
\end{aligned}
$$

Note that there are two different transformations according to the two different types of poles that the solutions of (1) can have (simple poles with residues 1 or -1$)$. The changes of variables $(2)$ are what we will call regularising transformations for the equation at points where the dependent variable becomes infinite. In this case, the transformations are known since Painlevé and can be found by the method of truncation. For the other Painlevé equations similar changes of variables are known.

Moving on to equations with a more complicated singularity structure, Filipuk and Halburd [5] considered a class of equations

$$
y^{\prime \prime}(z)=\sum_{k=1}^{N} a_{k}(z) y(z)^{k},
$$

with in general movable algebraic singularities and which includes the Painlevé equations $P_{I}$ and $P_{I I}$. Special cases of equations in this class had been studied earlier by Shimomura $[17,18]$. For equations in this class regularising transformations are found by means of an auxiliary function $W$ that remains bounded as a movable singularity is approached and which serves as an approximate 
first integral. The form of $W$ in [5] is prescribed as

$$
W(z)=y^{\prime}(z)^{2}+\sum_{k=1}^{N-1} \frac{b_{k}(z)}{y(z)^{k}} y^{\prime}(z)-2 \sum_{k=1}^{N+1} \frac{a_{k-1}(z)}{k} y(z)^{k},
$$

where the coefficient functions $b_{k}(z), k=1, \ldots, N-1$, are determined in terms of the functions $a_{k}(z)$ in the differential equation (4). Employing this auxiliary function Filipuk and Halburd wrote down a system of equations in certain variables $(u, v)$ which remains finite at the movable singularities of a solution and which becomes a regular initial value problem when the independent variable $z$ is interchanged with one of the dependent variables.

This class of equations (along with other classes which can be written in a Hamiltonian form), for which the only movable singularities of their solutions in the complex plane are algebraic branch points, was also revisited in [13] where the method of blowing up points of indeterminacy is applied. After the final blow-up of an equation, a system of equations which forms a regular initial value problem at the singular points where the solution in the original variables tends to $\infty$ was obtained (after interchanging the variables). Keeping track of the changes of variables introduced by the blow-ups one can find a bi-rational regularising transformation for the equations under consideration, from which the behaviour at the singularities of the solutions of the original equation follows.

Further classes of equations with movable algebraic singularities were treated by Filipuk and Halburd [6,7], who studied equations of Liénard type,

$$
y^{\prime \prime}=F(z, y) y^{\prime}+G(z, y),
$$

where $F$ and $G$ are polynomials in $y$ with analytic coefficients, where $\operatorname{deg}_{y} G \leq$ $n+1$ if $\operatorname{deg}_{y} F=n$. Similar to the case of equation (4), the proof that all movable singularities of solutions of equations in the class (5), obtained by analytic continuation of a local analytic solutions along a finite length curve, are algebraic branch points, makes use of an approximate first integral which in this case has the form

$$
W(z)=y^{\prime}(z)-\sum_{k=0}^{n} \frac{f_{k}(z)}{j+1} y(z)^{j+1}+\sum_{l=0}^{n} a_{l}(z) y(z)^{-l+1},
$$

where the functions $a_{l}(z)$ need to be determined in terms of the coefficient functions $f_{k}(z), k=0, \ldots, n$, and $g_{k}(z), k=0, \ldots, n+1$.

A class of Hamiltonian systems with movable algebraic singularities,

$$
q^{\prime}=\frac{\partial H}{\partial p}, \quad p^{\prime}=-\frac{\partial H}{\partial q},
$$

was studied in [12]. The proofs in all these papers that the solutions of the equations in the respective classes have only movable algebraic singularities all rely on an approximate first integral $W$ specified for each class separately. 
In the following we will consider a system of equations of the general form

$$
p^{\prime}=\frac{P_{1}(z, p, q)}{Q_{1}(z, p, q)}, \quad q^{\prime}=\frac{P_{2}(z, p, q)}{Q_{2}(z, p, q)},
$$

where $P_{i}$ and $Q_{i}, i=1,2$, are polynomials in $p$ and $q$ with coefficients rational in $z$, such that the fractions on the right hand side are in reduces terms, i.e. $P_{i}$ and $Q_{i}$ have no common factors in $\mathbb{C}(z)[p, q]$. Originally, the notion of a blowup, a fundamental type of bi-rational transformation, comes from algebraic geometry. It can be performed to resolve the singularities of an algebraic curve in projective space. By a deep and general theorem by Hironaka, the singular points of an algebraic curve can be completely resolved by a finite sequence of blow-ups. The space considered in the context of complex differential equations is the space of dependent variables $(p, q) \in \mathbb{C}^{2}$ of our system of equations (7). The equations $P_{i}(z, p, q) \equiv 0$ and $Q_{i}(z, p, q) \equiv 0$ define curves in the two-dimensional affine space over the field $\mathbb{C}(z)$. The points of intersection $P_{i}(z, p, q)=Q_{i}(z, p, q) \equiv 0$ for $i=1$ or $i=2$, where the right hand side of $(7)$ becomes indeterminate, are called the base points of the system. The aim is to remove these indeterminacies by some suitable bi-rational transformation in $p$ and $q$ which will be obtained by a series of blow-ups described in the following. Geometrically, the blow-up procedure separates out the lines through base points according to their slopes and, hence, adds a projective line to the space, which is called an exceptional divisor. The blow-up at a point $(p, q)=(a, b)$, where $a=a(z)$ and $b=b(z)$ can in general be rational functions in $z$, is defined by the following construction. One introduces new coordinate charts, $p=a+u=a+U V$ and $q=b+u v=b+V$ and re-writes the system in new coordinates $(u, v)$ and $(U, V)$. The exceptional line then corresponds to $u=0$ or $V=0$. As a simple example let us consider the blow-up of the system of equations

$$
y_{1}^{\prime}=\frac{y_{2}\left(y_{2}-a\right)}{y_{1}}, \quad y_{2}^{\prime}=y_{1} y_{2},
$$

where $a \neq 0$. Here $y_{1}=p, y_{2}=q$. We see from the first equation that the base points are $\left(y_{1}, y_{2}\right)=(0,0)$ and $\left(y_{1}, y_{2}\right)=(0, a)$. Blowing up the point $\left(y_{1}, y_{2}\right)=(0, a)$, we get two new systems

$$
u^{\prime}=A v+u v^{2}, \quad v^{\prime}=\frac{a u+u^{2} v-a v^{2}-u v^{3}}{u}
$$

and

$$
U^{\prime}=\frac{a+V-a U^{3} V-U^{3} V^{2}}{U V}, \quad V^{\prime}=U V(a+V) .
$$

We see from the first system that there is another base point $(u, v)=(0,0)$. On the contrary, each right-hand side of the $(U, V)$ system is either regular or 
of the form

$$
\frac{\lambda+f(z, U, V)}{g(z, U, V)},
$$

where $\lambda \neq 0$ and $f, g$ are polynomials in $U$ and $V$ with rational functions in $z$ as coefficients. On the exceptional curve, parametrised by $(U, V)=(c, 0), c \in \mathbb{C}$, we find that $f(z, c, 0)=g(z, c, 0) \equiv 0$, so although there is no indeterminacy, still the right-hand side becomes infinite on the exceptional curve introduced by the blow-up. However, starting from initial conditions $\left(U\left(z_{0}\right), V\left(z_{0}\right)\right)$, with $V\left(z_{0}\right) \neq 0$, the solution can never pass through a point $(U, V)=(c, 0)$ as the vector field $\left(U^{\prime}, V^{\prime}\right)$ becomes infinite and tangent to the exceptional curve. The only point on the exceptional curve a solution can pass through is the point $(u, v)=(0,0)$ in the first chart after the blow-up, which is also the only point of indeterminacy of the system $(u, v)$ and which requires blowing up further. We will continue to perform blow-ups of all base points until the right hand sides of the emerging systems are either of the form with the right-hand sides (8) or they are regular on the exceptional curve (in case we can resolve all singularities after a finite number of blow-ups). In the latter case, the system forms a regular initial value problem for initial data on the exceptional curve and hence has a local analytic solution in a neighbourhood of such point, as is the case for the Painlevé equations. For most of the equations considered in this article, the sequence of blow-ups does not terminate and we argue that this is an indicator of a more complicated structure of singularities. The question arises how, for a given system of equations of the form (7), whether or not the sequence of blowing up points of indeterminacy terminates.

\section{An Algorithm for the Singularity Structure of an Equation}

In order to study singularities of a given system of equations, we need to consider the system over $\mathbb{P}^{2}$ or $\mathbb{P}^{1} \times \mathbb{P}^{1}$. In this paper we choose the compactification $\mathbb{P}^{1} \times \mathbb{P}^{1}$. This is needed if we want to study what happens when one or two of the dependent variables of the system become infinite. The aim is to obtain the space of initial conditions for the system after a sequence of blowups, when it terminates, which then allows us to read off the different types of movable singularities that can occur in a solution. We will now formalise this process to be used as an algorithm. In the example of Smith's equation that follows we shall see that the algorithm does not necessarily terminate and at present we have no criterion to decide, for a given equation or a system, whether or not it will terminate. However, in case the algorithms finishes after a finite number of blow-ups for each of a finite number of base points, it allows us to obtain a complete list of different types of movable singularities that can occur in the solutions of an equation.

Given a system of two first-order differential equations,

$$
y_{1}^{\prime}=R_{1}\left(z, y_{1}, y_{2}\right), \quad y_{2}^{\prime}=R_{2}\left(z, y_{1}, y_{2}\right),
$$


we now formalise the process of finding the singularity structure of the solutions.

- Consider a rational compactification $\mathcal{P}$ of the space of dependent variables, $\left(y_{1}(z), y_{2}(z)\right) \in \mathbb{C}^{2}$, covered by a finite number of charts $\phi_{i}: \mathcal{P} \rightarrow$ $\mathbb{C}^{2}$. Consider the equations obtained by re-writing the system of equations in each chart, together with the original one.

- In this compact space the system of equations may possess a finite number of base points. If not we are already finished and the solutions can in fact be meromorphically continued in the whole complex plane without the fixed singularities. Otherwise we perform, for each base point, a sequence of blow-ups in some chart that contains the point. After every blow-up, one of the following situations will occur.

(a) The exceptional curve introduced by the blow-up, contains a finite number of new base points. By this we mean base points that are not the transform of some base point present in any of the other charts. Some of the new base points $(u, v)=(0, c)$ or $(U, V)=(1 / c, 0)$, where $c \neq 0$, are seen in both charts after the blow-up, but some $((u, v)=(0,0)$ or $(U, V)=(0,0))$ are seen only in one of the new coordinate charts.

(b) The system of equations in both charts after the blow-up is of the form

$$
u_{1}^{\prime}=\frac{\lambda+f_{1}\left(z, u_{1}, u_{2}\right)}{g_{1}\left(z, u_{1}, u_{2}\right)}, \quad u_{2}^{\prime}=\frac{\mu+f_{2}\left(z, u_{1}, u_{2}\right)}{g_{2}\left(z, u_{1}, u_{2}\right)}, \quad(\lambda, \mu) \neq(0,0) .
$$

The right hand side of at least one of the equations becomes infinite on the exceptional curve. Then an analytic continuation of any solution cannot pass through the exceptional curve. The sequence of blow-ups for the base point finishes. Following [13], we might further study the question of the presence of movable algebraic singularities, if the system can be regularized after interchanging the dependent and independent variables.

(c) The system of equations is regular on the exceptional curve. Also here, the sequence of blow-ups finishes and the solution can cross the exceptional curve. At such a point the solution is analytic in the chart after the last blow-up. The solution can thus be transformed by a sequence of bi-rational transformations back to the original variables $\left(y_{1}, y_{2}\right)$ which then either has a pole or is analytic.

\section{An Example by Painlevé}

In the previous sections we discussed the difference between an equation passing the Painlevé test and having the Painlevé property. To make this fact 
explicit, Painlevé constructed the example

$$
y^{\prime \prime}=\frac{2 y-1}{y^{2}+1}\left(y^{\prime}\right)^{2},
$$

for which one can obtain, about every point $z_{0} \in \mathbb{C}$, a one-parameter family of Laurent series expansions

$$
y(z)=\sum_{k=-1}^{\infty} c_{k}\left(z-z_{0}\right)^{k} .
$$

Indeed, substituting the series (11) into (10) one can find that $c_{-1}$ is arbitrary, $c_{0}=-1 / 2, c_{1}=-5 /\left(12 c_{-1}\right), c_{2}=-5 /\left(24 c_{-1}\right)$ and so on. Equation (10) is also invariant under $y \rightarrow-1 / y$. However, as one can check easily, the general solution of the equation is given by

$$
y(z)=\tan (\log (a z+b)),
$$

having simple poles at the points where the argument of the tan becomes an odd multiple of $\frac{\pi}{2}$ according to the Laurent series (11). The logarithmic singularity at the point $z_{0}=-\frac{b}{a}$ is not discovered through the Painlevé analysis.

We will show in the remainder of this section how the singularity structure of equation (10) can be understood when we apply the procedure of blow-ups. Therefore we first re-write the equation as an equivalent system in two variables which is more convenient for our analysis,

$$
\begin{aligned}
& y_{1}^{\prime}=\left(y_{1}^{3}+y_{1}\right) y_{2}, \\
& y_{2}^{\prime}=-\left(y_{1}^{2}+y_{1}+1\right) y_{2}^{2} .
\end{aligned}
$$

The reader may check that $y=y_{1}$ indeed satisfies the equation (10). We will now re-write this system in the other coodinate charts in $\mathbb{P}^{1} \times \mathbb{P}^{1}$. We shall take $Y_{1}=1 / y_{1}$ and $Y_{2}=1 / y_{2}$. In the $\left(Y_{1}, y_{2}\right)$ chart the system (13) becomes

$$
\begin{aligned}
Y_{1}^{\prime} & =-\frac{y_{2}\left(1+Y_{1}^{2}\right)}{Y_{1}}, \\
y_{2}^{\prime} & =-\frac{y_{2}^{2}\left(1+Y_{1}+Y_{1}^{2}\right)}{Y_{1}^{2}} .
\end{aligned}
$$

We see that we have the first base point $p_{1}=\left(Y_{1}=0, y_{2}=0\right)$. Re-writing the system in the $\left(y_{1}, Y_{2}\right)$ coordinate chart, we get

$$
\begin{aligned}
y_{1}^{\prime} & =\frac{y_{1}+y_{1}^{3}}{Y_{2}}, \\
Y_{2}^{\prime} & =1+y_{1}+y_{1}^{2} .
\end{aligned}
$$

This further gives the following base points: $p_{2}=\left(y_{1}=0, Y_{2}=0\right), p_{3}=$ $\left(y_{1}=-i, Y_{2}=0\right)$ and $p_{4}=\left(y_{1}=i, Y_{2}=0\right)$, where $i^{2}=-1$. Actually, if we compactify differently our system and consider the coordinate charts over $\mathbb{P}^{2}$, then the points $p_{3}$ and $p_{4}$ are not present. The final system in $\left(Y_{1}, Y_{2}\right)$ coordinates does not give any new base points. In the following we shall deal 
with only $p_{1}$ and $p_{2}$ points, which can be resolved after one blow-up, as the cascades of $p_{3}$ and $p_{4}$ seem to be infinite. Actually, the points $p_{3}$ and $p_{4}$ are singular values of $y$ in (10), so we will not consider them in the following. However, we shall fully understand the singularity structure from blowing up the first two base points.

First consider the system for $\left(Y_{1}, y_{2}\right)$. Performing the blow-up of the point $p_{1}$ introduces two new coordinate charts which we denote by $\left(u_{1}, v_{1}\right)$ and $\left(U_{1}, V_{1}\right)$. In these coordinates, the system of equation becomes

$$
\begin{aligned}
& u_{1}^{\prime}=-v_{1}-u_{1}^{2} v_{1}, \quad v_{1}^{\prime}=-v_{1}^{2}, \\
& U_{1}^{\prime}=1, \quad V_{1}^{\prime}=-\frac{1+U_{1} V_{1}+U_{1} V_{1}^{2}}{U_{1}^{2}} .
\end{aligned}
$$

We note that in both systems, one of the equations contains only one variable, so it can be solved explicitly. Let us take system (16). The second equation can be solved explicitly and we get $v_{1}=1 /(z-a)$, where $a$ is an arbitrary constant. The first equation is then a Riccati equation in $u_{1}$ given by

$$
u_{1}^{\prime}=-\frac{u_{1}^{2}+1}{z-a} .
$$

This equation can be easily solved and we get solution involving tan and log in the form $u_{1}=\tan (k-\log (a-z))$, where $k$ is arbitrary, which, after returning to original variables $y_{1}=1 / u_{1}$, is similar to (12). On the other hand, we know that the Riccati equation can be linearized. Indeed, by taking $u_{1}=(z-a) w^{\prime} / w$, where $w$ is a new function of $z$, we get

$$
w^{\prime \prime}+\frac{w^{\prime}}{z-a}+\frac{w}{(z-a)^{2}}=0 .
$$

This equation is Fuchsian at any point $z=a$. By performing the usual analysis of the Fuchsian equation, by substituting $w(z)=(z-a)^{r}$, we see that the indicial equation is $r^{2}=-1$ which gives indices $r= \pm i, i^{2}=-1$. Further, by taking $w(z)=(z-a)^{i}\left(b_{0}+b_{1}(z-a)^{i+s}\right)$, we find that the resonances satisfy $-s= \pm 3 i$. Indeed, linear equation (18) has a general solution

$$
w(z)=k_{1}(z-a)^{i}+k_{2}(z-a)^{-i}, \quad i^{2}=-1,
$$

where $k_{1}$ and $k_{2}$ are arbitrary constants, which gives solution (12) written in a slightly different but equivalent way. Thus, after the first blowup we immediately get the general solution. System (17) is analogous. From the first equation we can get that $U_{1}=t+a$ and a Riccati equation for the function $V_{1}$ which leads to a similar linear equation (18).

Now let us explain how expansion (11) arises from system (16). We can search for expansions of system (16) in the form $u_{1}=\sum_{j=0}^{\infty} a_{j}\left(z-z_{0}\right)^{j}, v_{1}=$ $\sum_{j=0}^{\infty} b_{j}\left(z-z_{0}\right)^{j}$ which gives $b_{1}=-b_{0}^{2}, a_{1}=-b_{0}-a_{0}^{2} b_{0}, b_{2}=b_{0}^{3}, a_{2}=\left(1+2 a_{0}+\right.$ $\left.a_{0}^{2}+2 a_{0}^{3}\right) b_{0}^{2} / 2$ and so on. Returning to original variables, we get an expansion $y_{1}(z)=1 / a_{0}+\left(b_{0}+a_{0}^{2} b_{0}\right)\left(z-z_{0}\right) / a_{0}^{2}+O\left(\left(z-z_{0}\right)^{2}\right)$, which is holomorphic at $z=z_{0}$. However, if we search for expansion of system (16) in the form 
$u_{1}=\sum_{j=1}^{\infty} a_{j}\left(z-z_{0}\right)^{j}, \quad v_{1}=\sum_{j=0}^{\infty} b_{j}\left(z-z_{0}\right)^{j}$, which crosses the exceptional curve parametrized by $b_{0}$, we get $b_{1}=-b_{0}^{2}, a_{1}=-b_{0}, a_{2}=b_{0}^{2} / 2, b_{2}=b_{0}^{3}$, $a_{3}=-2 b_{0}^{3} / 3, b_{3}=-b_{0}^{4}, a_{4}=3 b_{0}^{4} / 4$ and, returning to original variables, we get $y_{1}=-1 /\left(b_{0}\left(z-z_{0}\right)\right)-1 / 2+5 b_{0}\left(z-z_{0}\right) / 12-5 b_{0}^{2}\left(z-z_{0}\right)^{2} / 24+O\left(\left(z-z_{0}\right)^{3}\right)$, which is essentially, expansion (11).

Blowing up the point $p_{2}$ is very similar. Indeed, we get two new systems in the coordinate charts $\left(u_{2}, v_{2}\right)$ and $\left(U_{2}, V_{2}\right)$

$$
u_{2}^{\prime}=\frac{u_{2}^{2}+1}{v_{2}}, \quad v_{2}^{\prime}=1
$$

and

$$
U_{2}^{\prime}=-U_{2}^{2}, \quad V_{2}^{\prime}=1+U_{2} V_{2}+U_{2}^{2} V_{2}^{2},
$$

which can be analysed similarly. They can, in fact, be integrated by elementary methods as above. We observe that there are no further indeterminacies on the right hand sides of these systems. Thus, the resolution of points $p_{1}$ and $p_{2}$ leads to a complete explanation of the movable singularities of the original equation.

\section{Smith's Example}

For the Painlevé equations the process of repeatedly blowing up base points leads to a space of initial conditions for the dependent variables after a finite number of steps which allows us to read off the possible behaviour of a solution at its movable singularities. For certain type of equations with algebraic singularities considered in [13], the process of blowing up base points is also finite, but in order to obtain the behaviour at movable singularities, one needs to interchange the dependent and independent variables after a final blowup. The question arises, whether this process always terminates, say, for any second-order differential equation. That the answer to this question is negative can be seen from the following example, proposed by Smith [19],

$$
y^{\prime \prime}+4 y^{3} y^{\prime}+y=0 .
$$

In general, Smith studied equations of the form

$$
y^{\prime \prime}+f(y) y^{\prime}+g(y)=P(z),
$$

where $y=y(z)$ and $f$ and $g$ are polynomials of degrees $n$ and $m$ respectively with $n>m$. He showed that any movable singularity of a solution of the equation, which can be obtained by analytic continuation along a finite length curve, is an algebraic branch point of the form

$$
y(z)=\sum_{k=-1}^{\infty} c_{k}\left(z-z_{0}\right)^{k / n}, \quad c_{-1} \neq 0 .
$$


For this, he re-wrote equation (23) in the form of the system by introducing new functions $y_{1}=y, y_{2}=y_{1}^{\prime}+F(y)$, where $F(y)=\int_{0}^{y} f(\eta) d \eta$. Clearly, from equation (23), $y_{2}^{\prime}=P(z)-g\left(y_{1}\right)$. Further, by introducing $u=1 / y_{1}$, Smith obtained a system of equations for $y_{2}(u)$ and $z(u)$ which is regular for $u=0$ with $z(0)=z_{0}$ and $y_{2}(0)$ also finite. Thus, $y_{2}$ and $z$ can be expanded in Taylor series in $u$ around $u=0$ and inverting the series one obtains expansion (24).

However, within the class of equations (23), there exist equations with solutions having non-isolated singularities. Such singularities are necessarily accumulations points of movable algebraic poles. An explicit example is equation (22). Smith described a solution to this equation with an explicit parametrisation in terms of Bessel functions for which there is a singularity obtained by an analytic continuation along a curve of infinite length. In particular, he re-wrote equation (22) as a system of equations $d z / d y_{2}=-1 / y_{1}$ and $d y_{1} / d y_{2}=\left(y_{1}^{4}-y_{2}\right) / y_{1}$ and noting that the second equation only involves the dependent variable $y_{1}$, he reduced it to the Bessel equation after a change of variables. He studied the behaviour of solutions as $z \rightarrow z_{0}$ and $y_{2}(z) \rightarrow \infty$ and showed that such a point cannot be an algebraic singularity. Further, he showed that this point is an accumulation point of movable algebraic singularities described previously and thus is itself of non-algebraic form. In fact, the behaviour of the solution in the vicinity of this point is very complicated as it would have to be described by extending the solution over a Riemann surface with an infinite number of sheets.

We will now see how the method of blowing up the base points for the equivalent system of equations, after compactifying the space of dependent variables, leads to a sequence of blow-ups that does not terminate. This might be an indicator of the presence of non-isolated singularities. Moreover, we conjecture that a similar behaviour will also be present in non-autonomous equations of Liénard type. Following Smith [19], let us study the system

$$
y_{1}^{\prime}=y_{2}-y_{1}^{4}, \quad y_{2}^{\prime}=-y_{1}
$$

First, let us show how to obtain expansion around an algebraic singularity. By introducing $Y_{1}=1 / y_{1}$, we get a system of equations

$$
Y_{1}^{\prime}=\frac{1-y_{2} Y_{1}^{4}}{Y_{1}^{2}}, \quad y_{2}^{\prime}=-\frac{1}{Y_{1}} .
$$

By interchanging the dependent and independent variables, we obtain the system for $z=z\left(Y_{1}\right)$ and $y_{2}\left(Y_{1}\right)$ given by

$$
\frac{d z}{d Y_{1}}=\frac{Y_{1}^{2}}{1-y_{2} Y_{1}^{4}}, \quad \frac{d y_{2}}{d Y_{1}}=-\frac{Y_{1}}{1-y_{2} Y_{1}^{4}} .
$$


Searching for expansions of the form $z\left(Y_{1}\right)=z_{0}+\sum_{j=1}^{\infty} a_{j} Y_{1}^{j}, p\left(Y_{1}\right)=p_{0}+$ $\sum_{j=1}^{\infty} b_{j} Y_{1}^{j}$, we can find the unknown coefficients $a_{j}$ and $b_{j}$ and finally obtain

$$
\begin{gathered}
z\left(Y_{1}\right)=z_{0}+\frac{Y_{1}^{3}}{3}+\frac{p_{0} Y_{1}^{7}}{7}-\frac{Y_{1}^{9}}{18}+o\left(Y_{1}^{9}\right), \\
y_{2}\left(Y_{1}\right)=p_{0}-\frac{Y_{1}^{2}}{2}-\frac{p_{0} Y_{1}^{6}}{6}+\frac{Y_{1}^{8}}{16}+o\left(Y_{1}^{8}\right) .
\end{gathered}
$$

Inverting the series, we obtain expansion of the form (24), in particular,

$$
\begin{aligned}
y(z)= & y_{1}(z)=\frac{c_{-1}}{\left(z-z_{0}\right)^{1 / 3}} \\
& -\frac{c_{-1}}{2}\left(z-z_{0}\right)^{5 / 3}+c_{3}\left(z-z_{0}\right)+o\left(z-z_{0}\right), \quad c_{-1}^{3}=1 / 3,
\end{aligned}
$$

where $c_{3}$ is arbitrary (this corresponds to the resonance). Also, in this case

$$
y_{2}(z)=\frac{7 c_{3}}{3}-\frac{3 c_{-1}}{2}\left(z-z_{0}\right)^{2 / 3}+o\left(\left(z-z_{0}\right)^{2 / 3}\right)
$$

Performing blow-ups of the system (25) after compactifying it to $\mathbb{P}^{1} \times \mathbb{P}^{1}$ with $Y_{1}=1 / y_{1}$ and $Y_{2}=1 / y_{2}$, we obtain the first base point $p_{1}=\left(Y_{1}=\right.$ $\left.0, Y_{2}=0\right)$. Then we get two more points $p_{2}=\left(u_{1}=0, v_{1}=0\right)$ and $p_{3}=\left(U_{1}=\right.$ $0, V_{1}=0$ ). The point $p_{2}$ gives an infinite cascade (which is actually periodic as we obtain sequences of length 6 with 5 points having coordinates $u_{j}=0, v_{j}=0$ and then $u_{6}=0, v_{6}=k$ with $|k|$ decreasing in each sequence). The point $p_{3}$ is resolved after one more blow-up. For instance, in the coordinates $\left(U_{3}, V_{3}\right)$, given by $Y_{1}=U_{3} V_{3}^{2}$ and $Y_{2}=V_{3}$, system (25) becomes

$$
U_{3}^{\prime}=\frac{1-2 U_{3}^{2} V_{3}^{5}-U_{3}^{4} V_{3}^{7}}{U_{3}^{2} V_{3}^{6}}, \quad V_{3}^{\prime}=\frac{1}{U_{3}} .
$$

According to our algorithm the system has no more base points. A similar system is obtained in the coordinates $\left(u_{3}, v_{3}\right)$. Both equations are then of the form (9). At $z=z_{0}$, if we assume expansion of the form (26), $U_{3} \sim\left(z-z_{0}\right)^{-7 / 3}$ and $V_{3} \sim\left(z-z_{0}\right)^{4 / 3}$, so $U_{3} \rightarrow \infty$ as $z \rightarrow z_{0}$. Thus, a transformation $U_{3}=y_{2}^{2} / y_{1}$, $V_{3}=1 / y_{2}$ can be regarded as a regularising transformation (as there are no further base points), but we have no regular initial value problem with the required properties. The point $Y_{1}=0, Y_{2}=0$ corresponds to the point $y_{1}=\infty$, $y_{2}=\infty$ in original variables, so this is an indicator that there is a singularity $z=z_{0}$ of non-algebraic type (as otherwise $y_{2}$ is finite).

Finally, we choose another equivalent system of equations. Although we do not obtain in that case expansion (26) by cleverly re-writing the system (since it will correspond to the base point), we still see an infinite cascade. Let us consider the system

$$
y_{1}^{\prime}=y_{2}, \quad y_{2}^{\prime}=-y_{1}-4 y_{2} y_{1}^{3} .
$$

Clearly, in this case for the algebraic singularity $y_{1} \sim\left(z-z_{0}\right)^{-1 / 3}, y_{2} \sim$ $\left(z-z_{0}\right)^{-4 / 3}$, hence an algebraic expansion would correspond to the point $Y_{1}=0$ 
and $Y_{2}=0$ as $z \rightarrow z_{0}$. However, this point is the base point which we need to blow up after compactifying the system $(28)$ to $\mathbb{P}^{1} \times \mathbb{P}^{1}$. After a finite number of blowups (9 in this case) there are no more base points and the algorithm stops. The bi-rational transformation $Y_{1}=U V^{2}$ an $Y_{2}=U^{4} V^{8}\left(U^{3} V^{7}-1\right)$ leads to the system of the form (9) (and, similarly in the $(u, v)$ coordinates, since $u=U V$ and $v=1 / U)$. However, $U \sim\left(z-z_{0}\right)^{7 / 3}$ and $V \sim\left(z-z_{0}\right)^{-1}$. There is another base point for system (28) after compactification, namely, the point with coordinates $Y_{1}=0$ and $y_{2}=0$. This base point gives rise to the infinite cascade, which is also periodic as in the case of the previous system with the only difference that in this case $|k|$ increases. Hence, we can conclude that the procedure of blowing up for both systems indicates the existence of more complicated singularities than algebraic ones.

\section{The Painlevé-Ince Equation}

The Painlevé-Ince equation is a member of the so-called Riccati hierarchy and it can be written in the operator form as $D\left(y^{\prime}+y^{2}\right)=0$, where the operator $D=d / d z+y$. In the usual form it is given by

$$
y^{\prime \prime}+3 y y^{\prime}+y^{3}=0 .
$$

When the Painlevé test is applied, it can be shown that it possesses both positive and negative resonances. We can obtain the following two Laurent expansions. The first one is with a positive resonance and $z_{0}$ is a movable pole:

$$
y(z)=\frac{1}{z-z_{0}}+c_{0}-c_{0}^{2}\left(z-z_{0}\right)+o\left(z-z_{0}\right) .
$$

For the second family with negative resonance one needs to search for the expansion in the form $y(z)=2\left(z-z_{0}\right)^{-1}+\sum_{j=2}^{\infty} c_{-j}\left(z-z_{0}\right)^{-j}$. As it is shown in [2], the general solution of (29) is given by

$$
y(z)=\frac{2 z-z_{1}-z_{2}}{\left(z-z_{1}\right)\left(z-z_{2}\right)} .
$$

Depending on the way it is expanded (in the punctured disk or in the complex plane without the disk) one can get both Laurent expansions above. This explains the presence of the negative resonance. In general, there have been many studies on the meaning of negative resonances in the Painlevé test and on the importance of information that they contain (see, for instance, $[2,8]$ and the references therein).

Let us consider the system

$$
y_{1}^{\prime}=y_{2}, \quad y_{2}^{\prime}=-3 y_{1} y_{2}-y_{1}^{3},
$$

which is equivalent to (29). Compactifying as before, we see that there is a base point $p_{1}$ at $Y_{1}=0$ and $Y_{2}=0$. As we proceed with blow-ups we see the point $p_{2}$ with $u_{1}=v_{1}=0$. Then the cascade of base points splits into two 
cascades. The first one, coming from the point $p_{3}$ with $u_{2}=0, v_{2}=-2$, is infinite. The second one, starting with the point $p_{4}$ with $u_{2}=0, v_{2}=-1$, is resolved after one further blow-up. The regular system is obtained by using the bi-rational transformation $Y_{1}=u_{4}$ and $Y_{2}=u_{4}^{2}\left(u_{4} v_{4}-1\right)$. It is given by

$$
u_{4}^{\prime}=\frac{1}{1-u_{4} v_{4}}, \quad v_{4}^{\prime}=\frac{u_{4} v_{4}^{3}}{u_{4} v_{4}-1} .
$$

On the exceptional curve $u_{4}=0$ it is regular, so we get Taylor expansions around $z=z_{0}$ with $u_{4}\left(z_{0}\right)=0$ and $v_{4}\left(z_{0}\right)=-2 c_{0}$ arbitrary. This will correspond to the Laurent expansion (30). The infinite cascade indicates that the singularities can be more complicated.

A very similar splittng cascade (we just have positive values in $v_{2}$ for both points) is observed if one studies another equivalent system

$$
y_{1}^{\prime}=y_{2}-3 y_{1}^{2} / 2, \quad y_{2}^{\prime}=-y_{1}^{3},
$$

so we omit the details. It is interesting to note that only the third system gives more information about the general solution to the Painlevé-Ince equation. Let us consider the system

$$
y_{1}^{\prime}=y_{2}-y_{1}^{2}, \quad y_{2}^{\prime}=-y_{1} y_{2} .
$$

There are two base points $p_{1}$ with $Y_{1}=0, y_{2}=0$ and $p_{2}$ with $Y_{1}=0, Y_{2}=0$. The first cascade is infinite and the second one regularises after one more blow-up. In particular, we obtain the system

$$
u_{2}^{\prime}=\frac{v_{2}-u_{2}}{v_{2}}, \quad v_{2}^{\prime}=1
$$

Thus, $v_{2}(z)=z-z_{0}$ and $u_{2}(z)=\left(z^{2}-2 z z_{0}-2 c\right) /\left(2\left(z-z_{0}\right)\right)$, where $c$ is arbitrary. With $y_{1}=1 / u_{2}$ and $y_{2}=1 /\left(u_{2} v_{2}\right)$ this means that we reproduced the general solution above (31).

We remark that instead of the standard Painlevé-Ince equation we can study the so called generalized Painlevé-Ince equation [1] given by $y^{\prime \prime}+\alpha y y^{\prime}+$ $\beta y^{3}=0$. The study of singularities of equivalent systems is very similar to the Painlevé-Ince example. However, for generic values of the parameters $\alpha$ and $\beta$ the sequence of blow-ups does not seem to terminate unless we pose some conditions on these parameters.

\section{Discussion and Future work}

For the Painlevé equations, the method of blowing up the space of dependent variables at points of indeterminacy leads to the space of initial conditions, which is uniformly foliated by the solutions as was shown by Okamoto. We have applied the method of blowing up base points to equations with more complicated singularities other than just poles as in the Painlevé case. By means of several examples, we have seen that a great deal of information about the singularities of the solutions of these equations can be obtained 
from the blow-up structure. We have formalised this procedure to obtain a list of all possible types of singularities in the solutions of a given equation. This is only possible, however, if the process described terminates, i.e. if for every base point the sequence of blow-ups is finite. With Smith's and Painlevé-Ince equations we have seen examples where the sequence of blow-ups is infinite. This then is a hint that the equation can have more complicated, non-isolated or other, singularities.

The proposed method of resolution of indeterminacies should be used to study singularities of non-linear second-order differential equations along with other methods. It is a good indicator for the presence of both algebraic and nonalgebraic singularities. However, the method seems to depend on the choice of system which is equivalent to a given non-linear second order differential equation, so sometimes we can get more information by choosing a particular system.

Finally, let us give a number of remarks about future work. The Smith and Painlevé-Ince examples fall into the class of Liénard-type equations. We plan to study general non-autonomous Liénard-type equations in more detail. We conjecture that solutions of these equations will also possess non-isolated and other type singularities.

In general, when we apply this method, we need to carefully check the systems that are obtained at each step, whether they are divergent on the exceptional divisor or not. If the system has parameters, then depending on the parameters, the number of cascades may be different, and also some infinite cascades for generic values of the parameters might terminate for some special choice of parameters, which makes the analysis very inconvenient. So the analysis of systems with parameters is more complicated. This can be demonstrated with the example of an equation by Hayman [3], given by

$$
y y^{\prime \prime}-\left(y^{\prime}\right)^{2}=\alpha y+\beta y^{\prime}+\gamma,
$$

where $\alpha, \beta$ and $\gamma$ are parameters (one can also study a similar equation when these are functions of $z$ but the analysis is somewhat more complicated). This equation, studied for constant coefficients, is not integrable for generic values of parameters, but some particular solutions are meromorphic. In [3] it is shown, by using the local series analysis combined with techniques of the Wiman-Valiron theory, that all meromorphic solutions are either polynomials or entire functions of order one. So for generic values of the parameters this equation always contains a particular meromoprhic solution. Moreover, the general solution is meromorphic if and only if $\alpha=\gamma=0$ or $\beta=0$. Solutions in these cases are given in terms of either polynomials or exponential functions (see Lemmas 2.1 and 2.2 in [3] for explicit expressions). We have studied the following system equivalent to equation (35):

$$
y_{1}^{\prime}=y_{2}, \quad y_{2}^{\prime}=\frac{\gamma+\beta y_{2}+y_{2}^{2}+\alpha y_{1}}{y_{1}} .
$$


For generic values of parameters we see three infinite cascades. However, if $\gamma=0$, then one of the cascades is finite. In case $\beta=0$ two cascades are finite. Since in this case according to [3] the general solution should be entire, we conjecture that at some step in the infinite cascade we should make a change of variables of the form $u \rightarrow e^{u}$, for one of the variables in the charts $(u, v)$ or $(U, V)$, after which the system regularizes and the sequence of blow-ups stops (perhaps already after the first blow-up). When $\alpha=\gamma=0$, we see two seemingly infinite cascades and one cascade is finite (actually it is solvable in the sense that $v_{2}^{\prime}=0$ and $u_{2}^{\prime}=-\beta+u_{2} v_{2}$ ). We also conjecture that the two infinite cascades regularize and stop after the exponential change of dependent variable. It is not fully clear at which step in the cascade we can make this change of variables, perhaps already after the first blow-up in both cascades. For generic values of parameters it may not be possible to terminate all the cascades after this change of variables, only one of them and this will likely correspond to a particular entire solution. For instance, for generic values of the parameters, the base point $p_{3}$ corresponds to $Y_{1}=0, Y_{2}=0$ and after the first blow-up we obtain the system

$$
u_{3}^{\prime}=-u_{3} / v_{3}, \quad v_{3}^{\prime}=-\beta u_{3} v_{3}-\alpha u_{3} v_{3}^{2}-\gamma u_{3}^{2} v_{3}^{2},
$$

so we see the next base point with $u_{3}=v_{3}=0$ (in the other chart $U_{3}, V_{3}$ we have polynomial right-hand sides of both equations, so no more base points). However, after the exponential change of variable for $u_{3}$, the system is regular, but this might also depend on how we choose our equivalent system. It might be interesting to find another equivalent system to the equation of Hayman to check these conjectures. We remark that we do not always have 3 cascades of base points, for instance when $\gamma=\beta^{2} / 4$, we have only two cascades and we conjecture that in one of the cascades the exponential change of variables after the first blow-up is needed to regularize it. When we repeat the calculations with non-constant coefficients, which are more involved in this case, the calculations look very similar, and there might be conditions (like resonance conditions) to be imposed on the coefficients $\alpha(z), \beta(z), \gamma(z)$. We conjecture that in this case the equation always has holomorphic solutions. Whether the general solution is holomorphic for some choice of the coefficients remains open. We leave the analysis of the non-autonomous Hayman and Liénard cases for future papers, where we plan to understand better how the singularities (or even the cases with entire solutions) correspond to termination/non-termination of the cascades for a given system of equations.

The other interesting class of equations is with algebroid solutions (see, for instance, [9] for the simplest case of 2-valued algebroid solutions for the second order equation with the quintic polynomial right-hand side). We have studied both the general case in [13] where we showed how to regularize the system after interchanging the dependent and independent variables after the last blow-up and the simplest algebroid case (which actually reduces to the fourth Painlevé equation after some quadratic transformation and scaling) and 
we do see the differences in the last blow-up in the resulting systems in the algebroid case like the absence of certain (odd or even) powers of the variables. It remains to show how the expansions for the algebroid case arise. Moreover, more examples are needed to answer the open question how to distinguish the cases with generic movable algebraic singularities and with algebroid solutions (general or particular).

\section{Acknowledgements}

GF acknowledges the support of the National Science Center (Poland) through the Grant OPUS 2017/25/B/BST1/00931.

Open Access. This article is licensed under a Creative Commons Attribution 4.0 International License, which permits use, sharing, adaptation, distribution and reproduction in any medium or format, as long as you give appropriate credit to the original author(s) and the source, provide a link to the Creative Commons licence, and indicate if changes were made. The images or other third party material in this article are included in the article's Creative Commons licence, unless indicated otherwise in a credit line to the material. If material is not included in the article's Creative Commons licence and your intended use is not permitted by statutory regulation or exceeds the permitted use, you will need to obtain permission directly from the copyright holder. To view a copy of this licence, visit http://creativecommons. org/licenses/by/4.0/.

\section{References}

[1] Abraham-Shrauner, B.: Hidden symmetries and linearization of the modified Painlevé-Ince equation. J. Math. Phys. 34, 4809 (1993)

[2] Andriopoulos, K., Leach, P.G.L.: An interpretaation of the presence of both positive and negative resonances in the singularity analysis. Phys. Lett. A 359, 199-203 (2006)

[3] Chiang, Y.M., Halburd, R.G.: On the meromorphic solutions of an equation of Hayman. J. Math. Anal. Appl. 281, 663-677 (2003)

[4] Duistermaat, J.J., Joshi, N.: Okamoto's space for the first Painlevé equation in Boutroux coordinates. Arch. Ration. Mech. Anal. 202, 707-785 (2011)

[5] Filipuk, G., Halburd, R.: Movable algebraic singularities of second-order ordinary differential equations. J. Math. Phys. 50, 023509 (2009)

[6] Filipuk, G., Halburd, R.: Rational ODEs with movable algebraic singularities. Stud. Appl. Math. 123, 17-36 (2009)

[7] Filipuk, G., Halburd, R.: Movable singularities of equations of Liénard type. Comput. Methods Funct. Theory 9, 551-563 (2009)

[8] Fordy, A., Pickering, A.: Analysing negative resonances in the Painlevé test. Phys. Lett. A 160, 347-354 (1991) 
[9] Halburd, R., Kecker, T.: Local and global finite branching of solutions of ordinary differential equations. In: Proceedings of the Workshop on Complex Analysis and its Applications to Differential and Functional Equations, pp. 57-78. Publications of the University of Eastern Finland. Reports and Studies in Forestry and Natural Sciences, vol. 14. The University of Eastern Finland, Faculty of Science and Forestry, Joensuu (2014)

[10] Hinkkanen, A., Laine, I.: Solutions of the first and second Painlevé equations are meromorphic. J. Anal. Math. 79, 345-77 (1999)

[11] Howes, P., Joshi, N.: Global asymptotics of the second Painlevé equation in Okamoto's space. Constr. Approx. 39, 11-41 (2014)

[12] Kecker, T.: Polynomial Hamiltonian systems with movable algebraic singularities. J. Anal. Math. 129, 197-218 (2016)

[13] Kecker, T., Filipuk, G.: Regularising transformations for complex differential equations with algebraic singularities, submitted. https://arxiv.org/abs/2101. 03432

[14] Kowalevski, S.: Sur le problème de la rotation d'un corps solide autour d'un point fixe. Acta Math. 12, 177-232 (1889)

[15] Okamoto, K.: Sur les feuilletages associés aux équations du second ordre à points critiques fixes de P. Painlevé. Japan. J. Math. (N.S.) 5, 1-79 (1979)

[16] Shimomura, S.: Proofs of the Painlevé property for all Painlevé equations. Jpn. J. Math. 29, 159-180 (2003)

[17] Shimomura, S.: A class of differential equations of PI-type with the quasiPainlevé property. Ann. Mat. Pura Appl. 186, 267-80 (2007)

[18] Shimomura, S.: Nonlinear differential equations of second Painlevé type with the quasi-Painlevé property along a rectifiable curve. Tohoku Math. J. 60, 581-595 (2008)

[19] Smith, R.A.: On the singularities in the complex plane of the solutions of $y^{\prime \prime}+$ $y^{\prime} f(y)+g(y)=P(x)$. Proc. Lond. Math. Soc. 3, 498-512 (1953)

Galina Filipuk

Institute of Mathematics

University of Warsaw

Banacha 2

Warsaw 02-097

Poland

e-mail: filipuk@mimuw.edu.pl

Thomas Kecker

School of Mathematics and Physics

University of Portsmouth

Lion Gate Building, Lion Terrace

Portsmouth PO1 3HF

UK

e-mail: thomas.kecker@port.ac.uk

Received: October 29, 2021. 
Accepted: December 5, 2021.

Publisher's Note Springer Nature remains neutral with regard to jurisdictional claims in published maps and institutional affiliations. 